\title{
Optical interferometry and Gaia parallaxes for a robust calibration of the Cepheid distance scale
}

\author{
Pierre Kervella ${ }^{1,2}$, Antoine Mérand ${ }^{3}$, Alexandre Gallenne ${ }^{4}$, \\ Boris Trahin $^{1}$, Simon Borgniet ${ }^{1}$, Grzegorz Pietrzynski ${ }^{5,7}$, \\ Nicolas Nardetto ${ }^{6}$ and Wolfgang Gieren ${ }^{7}$ \\ ${ }^{1}$ Unidad Mixta Internacional Franco-Chilena de Astronomía (CNRS UMI 3386), \\ Departamento de Astronomía, Universidad de Chile, Santiago, Chile \\ ${ }^{2}$ LESIA (UMR 8109), Observatoire de Paris, PSL Research University, CNRS, UPMC, Univ. \\ Paris-Diderot, 92195 Meudon, France, email: pierre.kervella@obspm.fr \\ ${ }^{3}$ European Southern Observatory, Karl-Schwarzschild-str. 2, D-85748 Garching, Germany. \\ ${ }^{4}$ European Southern Observatory, Alonso de Córdova 3107, Casilla 19001, Santiago 19, Chile. \\ ${ }^{5}$ Nicolaus Copernicus Astronomical Center, Polish Academy of Sciences, Warszawa, Poland \\ ${ }^{6}$ Laboratoire Lagrange, UMR7293, Université de Nice Sophia-Antipolis, CNRS, Observatoire \\ de la Côte d'Azur, 06000 Nice, France \\ ${ }^{7}$ Universidad de Concepción, Departamento de Astronomía, Casilla 160-C, Concepción, Chile

\begin{abstract}
We present the modeling tool we developed to incorporate multi-technique observations of Cepheids in a single pulsation model: the Spectro-Photo-Interferometry of Pulsating Stars (SPIPS). The combination of angular diameters from optical interferometry, radial velocities and photometry with the coming Gaia DR2 parallaxes of nearby Galactic Cepheids will soon enable us to calibrate the projection factor of the classical Parallax-of-Pulsation method. This will extend its applicability to Cepheids too distant for accurate Gaia parallax measurements, and allow us to precisely calibrate the Leavitt law's zero point. As an example application, we present the SPIPS model of the long-period Cepheid RS Pup that provides a measurement of its projection factor, using the independent distance estimated from its light echoes.
\end{abstract}

Keywords. stars: distances, stars: individual (RS Pup), stars: oscillations, stars: variables: Cepheids, cosmology: distance scale

\section{Introduction}

One century after the discovery of their Period-Luminosity relation (the Leavitt law) by Leavitt \& Pickering (1912), Cepheids are still the keystone of the empirical cosmic distance ladder. However, due to the large distances of Cepheids (particularly the longperiod pulsators), only relatively imprecise parallax measurements are available (e.g., from Hipparcos; van Leeuwen et al. 2007). As a result, the calibration of the zero point of the Leavitt law using Galactic Cepheids is insufficient accurate. Gaia's DR2 high accuracy parallaxes will bring a tremendous improvement, with distances to hundreds of individual Cepheids measured to a few percent accuracy. Alternatively, a classical avenue to measure the distances to individual Galactic and LMC Cepheids is the Parallax-ofPulsation (PoP) method, also known as the Baade-Wesselink (BW) technique. We here briefly present the SPIPS modeling tool that we developed to reproduce the classical observables of the pulsation of Cepheids (radial velocity, photometry, interferometry,...), and how we plan to use it in conjunction with the Gaia parallaxes to calibrate the PoP technique and eventually the Leavitt law. 

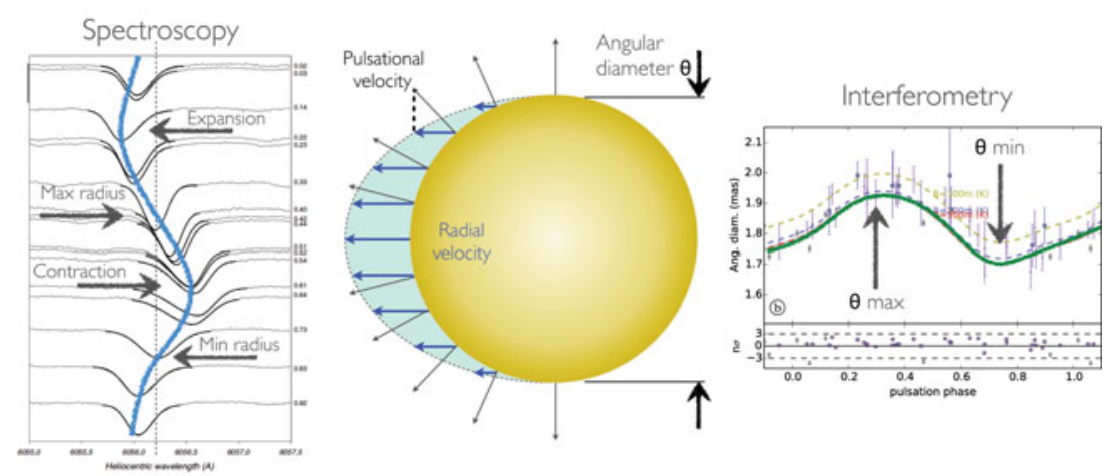

Figure 1. Principle of the classical Parallax-of-Pulsation distance determination on the Cepheid $\beta$ Dor. Left panel: Doppler displacement of the Fe I line at $6056 \AA$ obtained from high resolution HARPS spectra (Nardetto et al. 2006). Right panel: Interferometric angular diameter as a function of the pulsation phase (Breitfelder et al. 2016).

\section{The Parallax-of-Pulsation technique and SPIPS}

The PoP technique relies on the comparison of (1) the amplitude of the pulsation of the star from the integration of its radial velocity curve measured using spectroscopy and (2) the change in angular diameter estimated from its brightness and color, or measured using interferometry. The distance of the Cepheid is then obtained by fitting the linear and angular amplitudes (see, e.g., Storm et al. 2011). Although potentially precise at the percent level, the major weakness of the PoP technique is that it uses a numerical factor to convert disk-integrated radial velocities into photospheric velocities: the projection factor ( $p$-factor; Nardetto et al. 2017). It is defined as the ratio of the pulsational velocity over the radial velocity projected on the line of sight. The absolute accuracy of the PoP technique is currently limited by our knowledge of the $p$-factor, that is degenerate with the distance $d$ (the derived quantity is $d / p$ ). This parameter is particularly complex to model, as it represents simultaneously the spherical geometry, the limb darkening, and the gas dynamics in the Cepheid atmosphere.

The Spectro Photo Interferometry of Pulsating Stars (SPIPS; Mérand et al. 2015) model of a Cepheid is built assuming that the star is a radially pulsating sphere, for which the pulsational velocity $v_{\text {puls }}(t)$ and the effective temperature $T_{\text {eff }}(t)$ are the basic parameters. The cyclic variation of these parameters is represented using classical Fourier series or periodic splines functions. The photometry in all filters is computed using ATLAS9 atmosphere models, considering their bandpasses and zero points. The interstellar reddening is parametrized using the standard $E(B-V)$ color excess for Milky Way dust $\left(R_{V}=3.1\right)$ and computed for the phase-variable $T_{\text {eff }}$. Angular diameters including limb darkening are produced by the model to match the interferometric measurements, using SATLAS models (Neilson et al. 2013). Finally, circumstellar envelopes are included in the modeling (Gallenne et al. 2012; Gallenne et al. 2013), as well as the presence of stellar companions (Gallenne et al. 2015; Gallenne et al. 2017). SPIPS can be employed with only photometry and radial velocity, but interferometric angular diameters are independent of interstellar absorption, allowing to de-correlate the reddening and the effective temperature. Angular diameters thus bring a considerable added value to the quality and robustness of the derived parameters. Recent applications of SPIPS to Cepheids can be found in Mérand et al. (2015) ( $\delta$ Cep, $\eta$ Aql), Breitfelder et al. (2016) (Cepheids with HST/FGS parallaxes) and Breitfelder et al. (2015) (Type 2 Cepheid $\kappa$ Pav). 

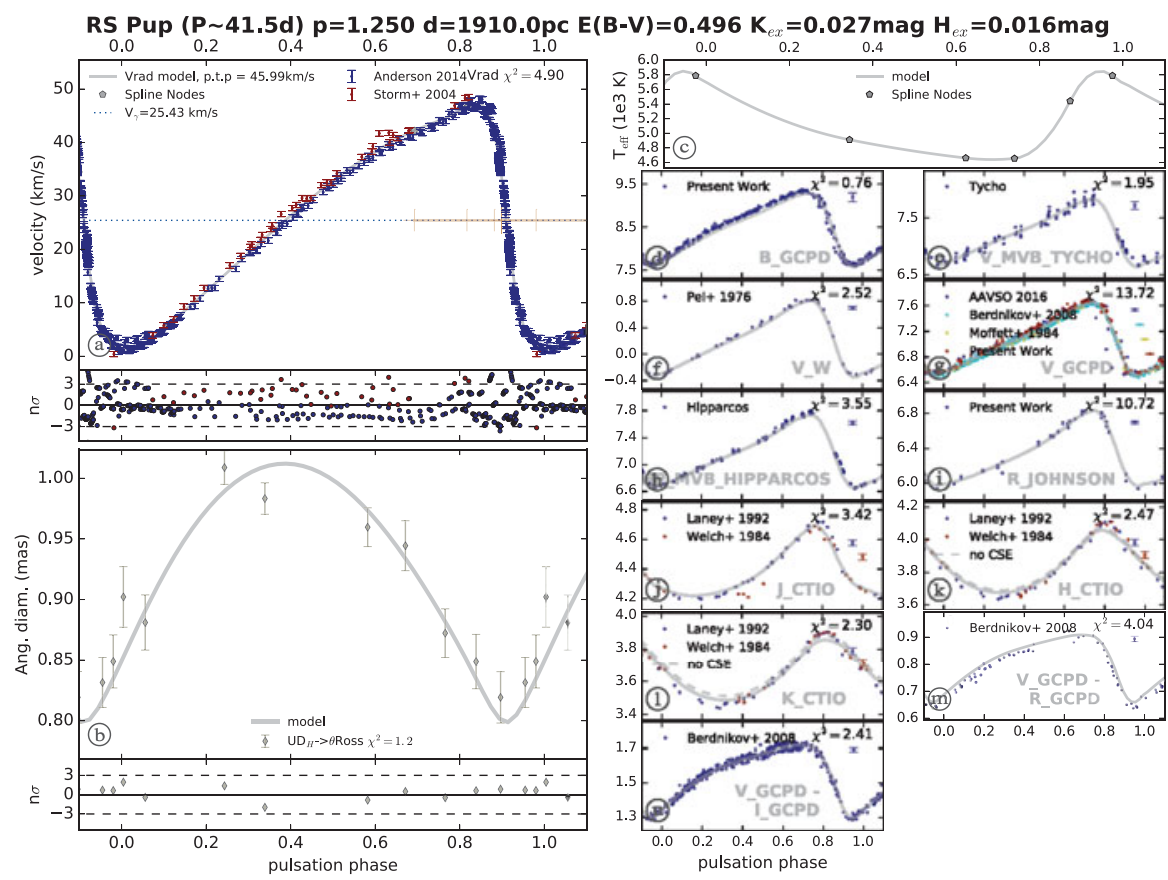

Figure 2. SPIPS combined fit of the observations of RS Puppis ( $P=41.5$ days). The radial velocity measurements are presented in the upper left panel, and the interferometric angular diameters in the lower left panel. The model's effective temperature is shown in the upper right panel, and the photometry in the different sub-panels of the right column (Kervella et al. 2017).

\section{The projection factor of $\mathbf{R S}$ Puppis and the $p-P$ relation}

RS Pup is one of the intrinsically brightest Cepheids in the Galaxy, and it is remarkable due to its large circumstellar nebula that reflects the light variations of the Cepheid. From the light echoes, Kervella et al. (2014) derived its distance of $d=1910 \pm 80$ pc. Knowing the distance resolves the intrinsic distance $/ p$-factor degeneracy of SPIPS and gives access to $p$. The pulsation model (Fig. 2) has been presented by Kervella et al. (2017), giving a best-fit $p$-factor of $p=1.25 \pm 0.06( \pm 5 \%)$. The addition of the $p$-factor of RS Pup to the limited set of existing $p$-factor measurements is particularly valuable, as it was up to now mostly limited to short and intermediate period Cepheids (Fig. 3). The other exception is $\ell$ Car $(P=35.5$ days $)$, whose parallax was measured by Benedict et al. (2007). The $p$-factors of $\ell$ Car and RS Pup are statistically identical within their uncertainties. The simple model of a constant $p=1.29 \pm 0.04$ over the sampled range of Cepheid periods (3.0 to 41.5 days) is consistent with the observations $\left(\chi_{\text {red }}^{2}=0.9\right.$; Kervella et al. 2017).

\section{The contribution of Gaia}

Gaia's DR2 in April 2018 will considerably improve the accuracy of Galactic Cepheid parallaxes compared to DR1 (Casertano et al. 2017; Gaia Collaboration 2017). Based on the foreseen accuracy, more than 400 Cepheid parallaxes will be measured with an accuracy $<3 \%$, out of which approximately one hundred to $<1 \%$. The observations of a sample of 18 long-period Cepheid using the spatial scanning mode of the HST/WFC3 by Casertano et al. (2016) will complement this data set. This unprecedented catalog will make it possible to obtain an extremely accurate calibration of the Leavitt law and other fundamental properties of Cepheids. However, to reach this accuracy implies that 


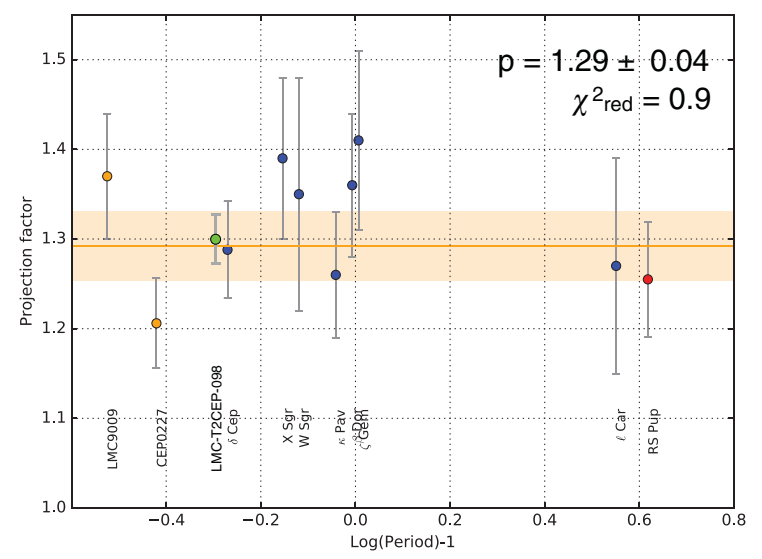

Figure 3. Measured p-factors of Cepheids (from Kervella et al. 2017). The green point is the measurement of the binary type 2 Cepheid OGLE-LMC-T2CEP-098 by Pilecki et al. (2017).

the pulsation models employed to interpret the observations must also reach this level of accuracy. The flexibility of the SPIPS modeling enables the integration of all types of new and archival observations in a consistent and robust approach. This will contribute to mitigate the influence of various systematics of observational origin, and bring the accuracy of the calibration of the Cepheid distance scale down to $1 \%$.

\section{Acknowledgements}

The authors acknowledge the support of the French Agence Nationale de la Recherche (ANR-15-CE31-0012-01 UnlockCepheids). W.G. and G.P. gratefully acknowledge the BASAL Centro de Astrofisica y Tecnologias Afines (CATA) PFB-06/2007. W.G. acknowledges support from the Chilean Millenium Institute of Astrophysics (MAS) IC120009. This research has received funding from the European Research Council (Horizon 2020 grant No 695099).

\section{References}

Benedict, G. F., McArthur, B. E., Feast, M. W., et al. 2007, AJ, 133, 1810

Breitfelder, J., Kervella, P., Mérand, A., et al. 2015 A\&SA, 576, A64

Breitfelder, J., Mérand, A., Kervella, P., et al. 2016, A\&\&A, 587, A117

Casertano, S., Riess, A. G., Anderson, J., et al. 2016, ApJ, 825, 11

Casertano, S., Riess, A. G., Bucciarelli, B., \& Lattanzi, M. G. 2017, A\& A, 599, A67

Gaia Collaboration, Clementini, G., Eyer, L., et al. 2017, $A \mathscr{E} A$, in press, arXiv:1705.00688

Gallenne, A., Mérand, A., \& Kervella, P. 2012, A\& A, 538, A24

Gallenne, A., Mérand, A., Kervella, P., et al. 2013, A\& $A$, 558, A140

Gallenne, A., Mérand, A., Kervella, P., et al. 2015, A\&A, 579, A68

Gallenne, A., Kervella, P., Mérand, A., Evans, N. R., \& Proffitt, C. 2017, these proceedings

Kervella, P., Bond, H. E., Cracraft, M., et al. 2014, A\& $A$, 572, A7

Kervella, P., Trahin, B., Bond, H. E., et al. 2017, A\& A, 600, A127

Leavitt, H. S. \& Pickering, E. C. 1912, Harvard College Observatory Circular, 173, 1

Mérand, A., Kervella, P., Breitfelder, J., et al. 2015, A\&A, 584, A80

Nardetto, N., Mourard, D., Kervella, P., et al. 2006, A\&A, 453, 309

Nardetto, N., Poretti, E., Rainer, M., et al. 2017, A\&A, 597, A73

Neilson, H. R. \& Lester, J. B. 2013, A\&̈A, 554, A98

Pilecki, B., Gieren, W., Smolec, R., et al. 2017, ApJ, in press (arXiv:1704.07782)

Storm, J., Gieren, W., Fouqué, P., et al. 2011, Aש\&A, 534, A94 